\title{
In vitro biofilm formation by methicillin susceptible and resistant Staphylococcus aureus strains isolated from cystic fibrosis patients
}

Antonietta Lambiase', Fabio Rossano', Mariassunta Del Pezzo', Valeria Raia², Giovanna Pulcrano', Massimiliano Marano', Domenica Cerbone ', Maria Rosaria Catania'

'Dipartimento di Biologia e Patologia Cellulare e Molecolare “Luigi Califano”, Facoltà di Medicina e Chirurgia, Università di Napoli "Federico II"

${ }^{2}$ Centro di Riferimento Regionale per la Fibrosi Cistica, Dipartimento di Pediatria, Facoltà di Medicina e Chirurgia, Università di Napoli "Federico II"

Key words: Fibrosi Cistica, Staphylococcus aureus meticillino-resistente, Biofilm

Formazione di biofilm in vitro da parte di ceppi di Staphylococcus aureus sensibili e resistenti alla meticillina isolati da pazienti con Fibrosi Cistica

\section{SUMMARY}

Staphylococcus aureus is one of the most common pathogens isolated from respiratory tracts of Cystic Fibrosis patients (CF). The infection by this pathogen starts in early infancy, often preceding chronic infections by Pseudomonas aeruginosa.

The infection and colonization by methicillin-resistant Staphylococcus aureus (MRSA) are, by then, events very frequent among CF patients and this bacterial isolation leads to complications in therapeutic management because of the limited treatment options.

Strains of Staphylococcus aureus are able to produce biofilms on natural or synthetic surfaces. Biofilms are sophisticated communities of matrix-encased bacteria and infections by biofilm-producing bacteria are particularly problematic because sessile bacteria can often withstand host immune responses and are generally much more tolerant to antibiotics.

The first aim of this work is to evaluate the ability of MRSA strains isolated from respiratory secretions of CF patients to develop biofilms in comparison with methicillin-sensitive Staphylococcus aureus (MSSA) strains obtained from respiratory secretions of CF patients. Therefore, our second aim is to evaluate the environmental influence on this ability.

To evaluate the development of biofilm on solid matrix and the possible environmental influence, we applied the method described by Christensen et al.

We found that a significantly higher number of MRSA strains were biofilm positive compared with MSSA strains $(p<0.05)$. The presence of glucose did not influence the ability to form biofilm in our MRSA strains $(p=0.165)$. MSSA strains are not strong biofilm-producers, but, when grown in TSB added with $0.25 \%$ glucose, the number of biofilm-forming strains increases, as expected.

These data suggest a possible association between methicillin-resistance and biofilm formation.

Received February 13, 2008

Accepted July 24, 2008

\section{INTRODUZIONE}

L'eziologia dell'infezione polmonare in Fibrosi Cistica (CF) è ascrivibile a diversi patogeni batterici ed esiste un preciso pattern batterico responsabile della malattia polmonare in CF, che ha, come caratteristica essenziale, l'essere età-specifico (12).

La prima infezione e colonizzazione in CF, che si manifesta in età infantile e pre-adolescenziale, vede come agenti eziologici Staphylococcus aureus ed Haemophilus influenzae. Successivamente, in età adolescenziale, subentra l'infezione da parte di Pseudomonas aeruginosa e poi, con il progredire dell'età, si verificano generalmente co-infezioni con altri batteri considerati opportunisti, in particolar modo Gram-negativi non fermentanti, come i batteri del Burkholderia cepacia complex (Bcc), Stenotrophomonas malto- 
philia, Alcaligenes xylosoxidans e Chryseobacterium spp (1, 4, 5, 10, 11, 12, 15). L'isolamento sempre più frequente di ceppi di Staphylococcus aureus resistenti alla meticillina (MRSA) da campioni respiratori di pazienti CF determina difficoltà terapeutiche e, conseguentemente, difficoltà di eradicazione del patogeno. La resistenza alla meticillina, e quindi a tutti i ß-lattamici, è correlata alla produzione di una penicillinbinding protein (PBP2) con attività trans-peptidasica necessaria alla sintesi del peptidoglicano anche in presenza di antibiotici ß-lattamici.

Molti patogeni isolati dal polmone CF sono connotati dall'abilità a formare biofilm, ossia comunità sessili di batteri che formano aggregati su superfici biologiche o sintetiche utilizzando una matrice polimerica di loro sintesi. Tale abilità conferisce vantaggi quali protezione dalla risposta immunitaria ed antibiotico-resistenza $(14,16)$.

Primo scopo del presente studio è analizzare la capacità di produzione di biofilm da parte di ceppi di Staphylococcus aureus sia sensibili (MSSA) che resistenti alla meticillina (MRSA) isolati da campioni respiratori di pazienti CF. Inoltre, il secondo scopo è confermare una possibile influenza da parte delle condizioni di crescita batterica sull'abilità nella produzione di biofilm (6, $7,9)$.

\section{MATERIALI E METODI}

\section{Ceppi batterici}

Gli isolati di Staphylococcus aureus utilizzati in questo studio sono ottenuti da colture di campioni di espettorato naturale o indotto, di pazienti afferenti al Centro di Riferimento per la CF della regione Campania, presso l’Università degli Studi di Napoli "Federico II".

Sono stati utilizzati ceppi di MSSA (n=30) e ceppi di MRSA $(n=30)$ isolati da pazienti in follow-up presso il Centro di Riferimento campano, collezionati nel periodo 2003-2006.

Colture dei microrganismi, analisi fenotipica e studio di antibiotico-resistenza

Per l'isolamento dei ceppi batterici, i campioni di espettorato naturale o indotto sono stati processati per le analisi batteriologiche routinarie.

In particolare, i campioni sono stati uniti ad un ugual volume di soluzione all'1\% di Ditiotritolo (Merck) per la fluidificazione e poi incubati a $37^{\circ} \mathrm{C}$ per 30 min. ed esaminati microscopicamente.

La semina per la ricerca di Staphylococcus aureus è fatta su terreno Agar Sangue. L'incubazione avviene in aerobiosi a temperatura di $37^{\circ} \mathrm{C}$ per 24 ore. Gli isolati ottenuti dall'esame colturale sono stati sottoposti a test di catalasi e coagulasi, e successivamente ad identificazione biochimica tramite il sistema automatico Phoenix (Becton Dickinson).

Per lo studio di antibiotico-resistenza è stato utilizzato un metodo in micro-diluizione, effettuato dallo stesso sistema utilizzato per l'identificazione. I ceppi di Staphylococcus aureus sono stati considerati resistenti alla meticillina se presentavano una MIC >2 nei confronti dell'oxacillina.

I criteri di sensibilità adottati rispettano i criteri interpretativi stabiliti dal National Committee for Clinical Laboratory Standards (13).

Formazione di biofilm

Per valutare l'aderenza batterica, abbiamo utilizzato un metodo spettrofotometrico (2) per cui gli inoculi batterici sono fatti crescere in TSB. Aliquote di tali inoculi sono poi diluite con TSB fresco (1:100) e $200 \mu \mathrm{L}$ di ogni inoculo sono posti in micro-piastre, incubate successivamente per 24 $\mathrm{h}$ a $37^{\circ} \mathrm{C}$. Dopo un lavaggio con PBS, i pozzetti sono colorati con cristal-violetto e l'aderenza alle superfici è letta allo spettrofotometro $(570 \mathrm{~nm})$. Le misurazioni di aderenza sono effettuate in triplicato. Infine i valori di lettura sono mediati.

In tal modo, i ceppi batterici sono stati infatti raggruppati in tre categorie:

1. Batteri non aderenti

2. Batteri debolmente aderenti

3. Batteri fortemente aderenti

secondo i seguenti cut-off:

1. OD $\leq 0.120 \rightarrow$ batteri non aderenti;

2. OD $>0.240 \rightarrow$ batteri fortemente aderenti;

3. OD $>0.120-0.240 \leq \rightarrow$ batteri debolmente aderenti

Per valutare l'influenza delle condizioni di crescita sull'abilità della produzione di biofilm, i ceppi batterici cresciuti in TSB sono poi diluiti con TSB addizionato con $0.25 \%$ di glucosio.

Successivamente si adotta la stessa procedura e gli stessi cut-off descritti precedentemente.

\section{Analisi statistica dei dati}

Al fine di valutare la significatività statistica dei dati ottenuti, i ceppi in studio sono stati divisi in quattro gruppi:

- Gruppo 1: ceppi MRSA coltivati in TSB

- Gruppo 2: ceppi MRSA coltivati in TSB+glucosio $0.25 \%$

- Gruppo 3: ceppi MSSA coltivati in TSB

- Gruppo 4: ceppi MSSA coltivati in TSB+glucosio $0.25 \%$

I dati in aderenza di ogni gruppo sono stati così confrontati tra loro attraverso analisi di varianza (ANOVA) per misure ripetute. Infine, sono stati considerati "statisticamente significativi" valori di $p$ inferiori a 0.05 . 


\section{RISULTATI}

I risultati ottenuti dalla tecnica applicata in tale studio (tabella 1 e 2) indicano che i ceppi di MRSA sono fortemente produttori di biofilm, indipendentemente dall'influenza che il glucosio può avere sulla crescita batterica (gruppo 1 vs gruppo $2, p=0.165$ ).

Dal confronto fra ceppi MRSA e ceppi MSSA risulta che i primi sono spiccatamente produttori di biofilm rispetto ai secondi (gruppo 1 vs gruppo 3, $p<<0.05$; gruppo 2 vs gruppo $3, p<<0.05$ ).

Inoltre i ceppi di MRSA sono maggiormente produttori di biofilm anche rispetto a ceppi di MSSA coltivati in mezzo addizionato con glucosio (gruppo 1 vs gruppo 4, $p<<0.05$; gruppo 2 vs gruppo 4 , $p<<0.05$ ).

I ceppi di MSSA, invece, non mostrano tale caratteristica (gruppo 3, 8/30 ceppi; 26.6\%).

Nel contempo, però, è molto interessante notare che, in terreno addizionato con glucosio, risulti statisticamente significativa la quota di ceppi MSSA che divengono produttori di biofilm (gruppo 3 vs gruppo $4, p<<0.05$ ).

\section{DISCUSSIONE}

L'incremento della sopravvivenza media dei pazienti CF è un dato correlabile sia alle nuove strategie terapeutiche adottate, sia alla disponibilità di centri di riferimento specializzati. Nel contempo, però, la pressione selettiva esercitata proprio dai trattamenti chemioterapici ha inevitabilmente dato la possibilità dell'emersione di ceppi con particolari resistenze.

Staphylococcus aureus rappresenta, insieme a Pseudomonas aeruginosa, uno dei maggiori patogeni responsabili di infezioni polmonari in CF e tale consistente contributo nello sviluppo della patologia infettiva polmonare in CF è proprio

Tabella I. Produzione di biofilm nei ceppi in studio

\begin{tabular}{|l|l|l|}
\hline Ceppi & Produzione di biofilm \\
\hline & Crescita in TSB & Crescita in TSB $+0.25 \%$ glucosio \\
\hline MRSA $n=30$ & $30(100 \%)$ (tutti fortemente aderenti) & $30(100 \%)$ (tutti fortemente aderenti) \\
\hline MSSA $n=30$ & $8(26.6 \%)$ (tutti debolmente aderenti) & $15(50 \%)(8$ debolmente aderenti, 7 fortemente aderenti) \\
\hline
\end{tabular}

Tabella 2. Valori di assorbanza (valore medio) da parte dei ceppi in studio

\begin{tabular}{|c|c|c|c|c|}
\hline Ceppo & $\begin{array}{l}\text { Produzione di biofilm } \\
\text { per MRSA (gruppo I) }\end{array}$ & $\begin{array}{c}\text { Produzione di biofilm } \\
\text { per MRSA } \\
\text { (TSB+0.25\% glucosio) } \\
\text { (gruppo2) }\end{array}$ & $\begin{array}{l}\text { Produzione di biofilm } \\
\text { per MSSA (gruppo 3) }\end{array}$ & $\begin{array}{c}\text { Produzione di biofilm } \\
\text { per MSSA } \\
\text { (TSB+0.25\% glucosio) } \\
\text { (gruppo 4) }\end{array}$ \\
\hline 1 & 0.31 & 0.31 & 0.012 & 0.010 \\
\hline 2 & 0.30 & 0.31 & 0.036 & 0.032 \\
\hline 3 & 0.30 & 0.30 & 0.10 & 0.20 \\
\hline 4 & 0.28 & 0.30 & 0.024 & 0.020 \\
\hline 5 & 0.31 & 0.31 & 0.01 & 0.015 \\
\hline 6 & 0.29 & 0.32 & 0.011 & 0.010 \\
\hline 7 & 0.30 & 0.30 & 0.09 & 0.09 \\
\hline 8 & 0.30 & 0.30 & 0.069 & 0.07 \\
\hline 9 & 0.32 & 0.31 & 0.047 & 0.20 \\
\hline 10 & 0.40 & 0.38 & 0.015 & 0.21 \\
\hline 11 & 0.32 & 0.38 & 0.014 & 0.21 \\
\hline 12 & 0.31 & 0.35 & 0.023 & 0.03 \\
\hline 13 & 0.29 & 0.31 & 0.056 & 0.03 \\
\hline 14 & 0.31 & 0.35 & 0.021 & 0.27 \\
\hline 15 & 0.37 & 0.32 & 0.022 & 0.03 \\
\hline 16 & 0.26 & 0.30 & 0.18 & 0.20 \\
\hline 17 & 0.32 & 0.31 & 0.039 & 0.03 \\
\hline 18 & 0.26 & 0.30 & 0.20 & 0.20 \\
\hline 19 & 0.35 & 0.32 & 0.23 & 0.28 \\
\hline 20 & 0.30 & 0.30 & 0.017 & 0.020 \\
\hline 21 & 0.31 & 0.30 & 0.011 & 0.018 \\
\hline 22 & 0.25 & 0.39 & 0.19 & 0.30 \\
\hline 23 & 0.36 & 0.32 & 0.20 & 0.21 \\
\hline 24 & 0.37 & 0.35 & 0.012 & 0.03 \\
\hline 25 & 0.25 & 0.25 & 0.21 & 0.30 \\
\hline 26 & 0.25 & 0.29 & 0.20 & 0.25 \\
\hline 27 & 0.33 & 0.32 & 0.02 & 0.10 \\
\hline 28 & 0.28 & 0.30 & 0.12 & 0.25 \\
\hline 29 & 0.32 & 0.30 & 0.10 & 0.20 \\
\hline 30 & 0.30 & 0.30 & 0.29 & 0.30 \\
\hline
\end{tabular}


ascrivibile alla capacità di produrre biofilm.

I dati mostrati indicano che ceppi di Staphylococcus aureus caratterizzati dalla resistenza alla meticillina sono, nel nostro caso, anche caratterizzati da una spiccata capacità di formare biofilm almeno in vitro. È noto che nella produzione di biofilm da parte di Staphylococcus aureus è coinvolto il locus genico di adesione intracellulare (ica operon) (3) così come per la resistenza alla meticillina sono coinvolte le cassette geniche mec (SCCmec tipo I, II, III) (8), ma non vi sono dati in letteratura riguardo una possibile ed eventuale correlazione anche biochimica fra resistenza alla meticillina in ceppi di Staphylococcus aureus produttori di biofilm. Ciò nonostante, i nostri dati sono suggestivi di una stringente correlazione, almeno in termini di prevalenza, fra queste due caratteristiche.

Inoltre, tali dati confermano anche che la complessa regolazione della formazione del biofilm dipende fortemente anche dalle condizioni ambientali.

In conclusione, i nostri dati indicano che lo studio quantitativo della produzione di biofilm da parte di ceppi di MRSA isolati dal polmone CF risulta essere un importante tassello nel complessivo studio fenotipico, rappresentando anch'esso uno strumento di conoscenza necessario per caratterizzare ulteriormente i ceppi responsabili di infezioni polmonari in pazienti CF.

\section{BIBLIOGRAFIA}

1. Armstrong DS, Grimwood K, Carlin JB, et al. Lower airway inflammation in infants and young children with cystic fibrosis. Am J Respir Crit Care Med 1997; 156: 1197-1204.

2. Christensen GD, Simpson WA, Younger JJ, et al. Adherence of coagulase-negative staphylococci to plastic tissue culture plates: a quantitative model for the adherence of staphylococci to medical devices. $J$ Clin Microbiol 1985; 22(6): 996-1006.

3. Cramton SE, Gerke Ch, Schnell NF, Nichols WW, Gotz F. The intercellular adhesion (ica) locus is present in Staphylococcus aureus and is required for biofilm formation. Infect Immun 1999; 67: 5427-33.
4. Cystic Fibrosis Foundation Patient Registry. 2001 Annual Data Report to the Center Directors. Bethesda, MD: Cystic Fibrosis Foundation; 2002.

5. Dakin CJ, Numa AH, Wang H, Morton JR, Vertzyas CC, Henry RL. Inflammation, infection, and pulmonary function in infants and young children with cystic fibrosis. Am J Respir Crit Care Med 2002; 165: 904-910.

6. Freeman DJ, Falkiner FR, Keane CT. New method for detecting slime production by coagulase negative sthaphylococci. J Clin Pathol 1989, 42 (8): 872-87.

7. Grinholc M, Weyrzyn G, Kurlenda J. Evaluation of biofilm production and prevalence of the icaD gene in methicillin-resistant and methicillin-susceptible Staphylococcus aureus strains isolated from patients with nosocomial infections and carriers. FEMS Immunol Med Microbiol 2007; 50 (3): 375-9.

8. Ito T, Katayama Y, Asada K, Mori N, Tsutsumimoto K, Tiensasitorn C, Hiramatsu K. Structural comparison of three types of Staphylococcus cassette chromosome mec integrated in Methicillin-resistant Staphylococcus aureus. Antimicrob Agents Chemother 2001; 45: 1326.

9. Knobloch JK, Horstkotte MA, Rohde H, Mack D. Evaluation of different detection methods of biofilm formation in Staphylococcus aureus. Med Microbiol Immunol 2002; 191 (2): 101-106

10. Lambiase A, Raia V, Del Pezzo M, Sepe A, Carnovale V, Rossano F. Microbiology airway disease in a cohort of patients with Cystic Fibrosis. BMC Infectious Diseases. 2006; 6(1): 4.

11. Lambiase A, Del Pezzo M, Raia V, Sepe A, Ferri P, Rossano F. Chryseobacyerium respiratory tract infections. J Infection 2007; 55 (6): 518-23.

12. Lyczak JB, Cannon CL, Pier GB. Lung infections associated with cystic fibrosis. Clin Microbiol Rev 2002; 15: 194-222.

13. National Committee for Clinical Laboratory Standards. Performance Standards for Antimicrobial Susceptibility testing. Approved standard M100-S14, NCCLS, Wayne, 2004

14. Prince A. Biofilms, antimicrobial resistance, and airway infection. N Engl J Med 2002; 347: 1110-1111.

15. Rosenfeld M, Gibson RL, McNamara S, et al. Early pulmonary infection, inflammation, and clinical outcomes in infants with cystic fibrosis. Pediatr Pulmonol 2001; 32: 356-366.

16. Singh PK, Schaefer AL, Parsek MR, Moninger TO, Welsh MJ, Greenberg EP. Quorum-sensing signals indicate that cystic fibrosis lungs are infected with bacterial biofilms. Nature 2000; 407: 762-764. 